\title{
Droit et Géographie
}

Construire une approche pour (re)lire l'environnement

Law and Geography. Towards an approach to read (over) environment

\section{Nadia Belaidi et Geneviève Koubi}

\section{(2) OpenEdition}

1 Journals

Édition électronique

URL : http://journals.openedition.org/developpementdurable/10798

DOI : 10.4000/developpementdurable.10798

ISSN : 1772-9971

Éditeur

Association DD\&T

\section{Référence électronique}

Nadia Belaidi et Geneviève Koubi, « Droit et Géographie », Développement durable et territoires [En ligne], Vol.6, n¹ | Mars 2015, mis en ligne le 31 mars 2015, consulté le 20 avril 2019. URL : http://

journals.openedition.org/developpementdurable/10798; DOI : 10.4000/developpementdurable.10798

Ce document a été généré automatiquement le 20 avril 2019.

\section{(c) (i) (8)}

Développement Durable et Territoires est mis à disposition selon les termes de la licence Creative Commons Attribution - Pas d'Utilisation Commerciale 4.0 International. 


\title{
Droit et Géographie
}

\author{
Construire une approche pour (re)lire l'environnement \\ Law and Geography. Towards an approach to read (over) environment
}

Nadia Belaidi et Geneviève Koubi

1 Ces dernières années, les sciences humaines et sociales ont connu un changement considérable dans leur appréhension du rôle de l'espace dans l'explication des processus sociaux. Ce spatial turn (tournant spatial) est fondé sur l'influence intensive que revêtent les facteurs spatiaux pour une appréhension rigoureuse de la complexité des relations sociales, en particulier au vu des transformations globales des rapports sociaux qui se sont produites ces trente dernières années. Dans les études socio-juridiques, cet intérêt pour les relations spatiales a suscité la création d'une interface interdisciplinaire (droit/ géographie) qui, en se saisissant du contexte spatial des phénomènes juridiques, interpelle le rôle du droit dans la reproduction des relations sociales ainsi que l'appui de la géographie pour la construction des normes juridiques.

2 Dans le monde anglo-saxon (nous y inclurons pour l'occasion le Canada français du fait de la circulation intellectuelle que la proximité géographique avec les États-Unis contribue à développer et l'ouvrage de Forest, 2009), on compte, dès le début des années 1990, un nombre significatif d'ouvrages, d'articles ou de numéros spéciaux de revues scientifiques sur la géographie des normes juridiques (et notamment Blomley, 1989, 1994 ; Urban Geography, 1990a , 1990b ; Delaney, 1993 ; Blomley et al., 2001; Holder et Harrison, 2003 ; Thom, 2004 ; Taylor, 2006 ; Braverman, 2011 ; Braverman et al. 2014) qui illustrent l'étendue, la portée et la profondeur de la recherche en Legal geography. Une partie significative de cette littérature a pour but d'établir des liens entre les préoccupations des études critiques du droit et les recherches en géographie critique tandis qu'un petit nombre d'auteurs réfléchit aux conséquences théoriques et méthodologiques du « tournant spatial » sur les études socio-juridiques. Cette démarche a pourtant assez peu touché, en France, la recherche en sciences juridiques.

3 C'est précisément cette combinaison entre droit et géographie et la manière qu'elle a d'exister et d'être discernée qui nous a intrigués ; elle nous a ainsi conduits à interroger ses présupposés et ses aboutissants. 
4 Le thème des relations entre la/les Géographie(s) et le/les Droit(s) invite à s'emparer des problématiques environnementales afin d'évaluer la «création» d'une approche spécifique pour analyser les questions environnementales au prisme des discours juridiques.

5 L'environnement est saisi par les géographes, principalement, par le biais du territoire, or le territoire demeure une notion implicite en droit. En nous penchant sur ces notions et sur cette relation, nous pouvons susciter l'apparition d'une approche spécifique des problématiques environnementales autour des concepts du droit et de la géographie à l'image de ce qui s'est construit aux États-Unis avec la Legal geography. Notre objectif n'est, toutefois, pas de transmuer des concepts anglo-saxons pour les transposer dans des systèmes détachés de la Common Law. Notre ambition est d'explorer les contours d'une démarche interdisciplinaire appliquée sur des terrains sociaux et à des espaces linguistiques autres que ceux qui l'ont faite émerger. Pour ce faire, encore fallait-il, sans prétendre à la représentativité et en restreignant la réflexion dans l'espace linguistique francophone, illustrer la diversité des cheminements. Aussi, nos observations, recherches et analyses ont-elles été travaillées durant le séminaire "Géographie et droit Géographie du droit » que nous avons tenu à trois reprises (les 17-18 septembre 2010, les 3-4 décembre 2010, les 28-29 octobre 2011) à Carcassonne.

6 Ce séminaire réunissait des géographes et des juristes de divers horizons. Son canevas initial reposait sur l'idée que la convergence de perspectives entre la Géographie et le Droit permet de lire le droit à partir des approches spatiales et avec les mots de la géographie et, réciproquement, d'appréhender le spatial avec les mots et la théorie du droit. Au-delà des seuls questionnements sur l'efficience du Droit ou sur les modes d'élaboration et la détermination des champs d'application des normes juridiques dans l'espace, sur les terrains et dans les territoires, notre ambition était, d'une part, de réfléchir conjointement à la construction des normes juridiques en matière d'environnement à partir des modèles géographiques et, d'autre part, de tenter de structurer la réflexion collective autour des modulations des assemblages entre droit et géographie : comment ces espaces et territoires (environnementaux) s'accordent-ils avec le système juridique?

7 La première rencontre s'est attachée aux formes discursives du Droit ; par-delà leur efficience quant à leur tendance à caractériser, délimiter, spécifier, différencier, singulariser ou particulariser les définitions, il s'agissait de repérer les modes d'élaboration et la détermination des champs d'application des normes juridiques dans l'espace, sur les terrains et dans les territoires. La deuxième rencontre, tout en poursuivant l'enrichissement mutuel des champs de recherches, a voulu dépasser les cadrages et les limites dans le but de réfléchir conjointement aux modes de construction des normes juridiques à partir des modèles géographiques. Ainsi, sans s'arrêter aux questions inhérentes à l'édification des territoires ou des espaces par le Droit, nous avions tenté de structurer la réflexion autour des fondations des assemblages entre droit et géographie : comment ces espaces et territoires s'accordent-ils avec le système juridique ? La troisième rencontre, enfin, avait pour objet de mettre en relation la façon dont chacun des participants envisageait la constitution d'un paradigme associant le droit et la géographie dans le cadre de leurs recherches respectives, selon des thématiques spécifiques théoriques ou pratiques (envisageant ainsi, par exemple, les questions relatives aux inondations, aux sites nucléaires, aux ports maritimes, aux parcs naturels nationaux ou internationaux, etc.). 
8 La relation entre le droit et la géographie dépendant du regard porté sur une thématique qui peut être aussi bien globale que locale, le présent dossier apparait donc comme le résultat des travaux menés lors du séminaire « Géographie et droit - Géographie du droit » dont il retient les différents axes. L'idée d'une convergence de perspectives entre la/les Géographie(s) et le(s) Droit(s), qui constitue le fil et le moteur de notre réflexion, est ordonnée autour d'une constante sociale et politique rarement étudiée - peut-être parce qu'elle semble à la fois "évidente» et "discordante». Cette liaison aux ajustements variables, assez expérimentale, a suscité de fructueux échanges lors du séminaire, lesquels ont rehaussé les contributions réunies en ce dossier et font regretter de ne pouvoir en délivrer toute la richesse et la diversité.

Notre postulat repose sur la conviction que le droit ne peut correctement être compris qu'à partir de l'analyse des relations qu'il entretient avec les systèmes sociaux, économiques, politiques et écologiques. Ainsi, il ne s'agit pas d'allouer une dimension géographique au droit ni même de simplement le situer dans son contexte géographique. Il s'agit plutôt de tenter d'identifier comment le droit et l'espace, au travers de la géographie, sont liés l'un à l'autre en mettant en relief les points de tension et les points de convergence.

10 Réciproquement " légaliser/juridiciser » la géographie aide à comprendre comment le discours du droit construit et légitime des espaces. En effet, le droit a lieu : la loi (au sens large) a une " présence physique " voir même de multiples présences. Cela permet de ne plus considérer cette loi comme un simple « mot». Ainsi libéré de son confinement, on peut observer le droit « au travail» dans un monde dominé par le physique. De ce fait, la posture tranche radicalement avec les approches juridique qui idéalisent un droit séparé de toute rationalité et réflexivité et le dépeignent comme imperméable aux influences matérielles, physiques, spatiales et culturelles. Un des principaux atouts de la prise en compte de la spatialité du droit est de démasquer les influences du positivisme sur les analyses du droit, y compris sur des institutions sociales informelles et des pratiques comme la communauté ou la coutume. Le positivisme a pour effet « de déstabiliser la normativité et l'objectivité du droit et ainsi de détruire son intention » (Jones, 2003, p. 186).

11 Il en découle une «théorie » où le droit et l'espace sont mutuellement constitutifs. Une théorie qui combine l'interdépendance du discours juridiques aux idées critiques en géographie humaine à propos de l'hétérogénéité de l'espace social. Certes Montesquieu, dans L'Esprit des lois, avait expliqué les constructions politiques par l'analyse des relations qu'entretiennent les ordres juridiques locaux et le cadre géographique ; mais c'est principalement au droit positif qu'il s'intéressait. Or notre posture cherche à dépasser le positivisme juridique notamment en ce qui concernent les distinctions considérées comme évidente : social/matériel, humain/non-humain, personnes/choses.

12 Ces trois points nous permettent d'accompagner le lecteur d'un bout à l'autre de notre réflexion en suivant une structure tripartite.

D'abord une réflexion centrée sur une relation Géographie(s) et Droit(s) «appliqués », une image_standard du droit saisi par la géographie comme le confirment les récentes manifestations ou publications sur la liaison de ces disciplines, pour certaines largement inspirées par notre séminaire. La démonstration débute avec la présentation, en guise de construction thématique, de la première réflexion en français sur l'approche interdisciplinaire abordée (P. Forest). Elle se poursuit par les positionnements du droit et 
de la géographie dans les questionnements environnementaux entre normes et terrains en examinant de quelle manière la mise en relation de la géographie et du droit fournit des explications sur le plan de la construction du droit dans le domaine des AOC et vise finalement la catégorisation (E. Rouvellac), puis dans les modalités de circulation à travers les paysages, renseigne sur la construction du patrimoine (S. de Carrara, Y.-F. Le Lay) et retrace les modes d'appréhension des relations conflictuelles de proximité (A. Azuela, P. Melé et V. Ugale).

Ensuite, puisque les terminologies détiennent une influence dans l'appréhension de cette relation pluridisciplinaire - les contributions précédentes prouvant combien nous avons des thèmes et problématiques partagées - l'ensemble se poursuit par une relation Géographie (s) et Droit(s) « discutés » à partir, d'une part, d'une réflexion sur des allégations lexicales dans l'espace (G. Koubi) et, d'autre part, de la (re)définition d'une notion et de son champs d'étude (N. Belaïdi).

Enfin, le dossier se termine par une réflexion sur les interrogations soulevées - le poil à gratter, sous forme d'un état des lieux de la question à partir d'une mise en lumière des questionnements initiaux (J.-F. Boudet).

\section{BIBLIOGRAPHIE}

"Law, Regulation and Geography I", 1990a, Urban Geography, special issue, volume 11, nº5.

"Law, Regulation and Geography II", 1990b, Urban Geography, special issue, volume 11, nº6.

Blomley N., 1989, "Text and context: Rethinking the law-space nexus", Progress in Human Geography, nº13, p. 512-534.

Blomley N., 1994, Law, space and the geographies of power, Guilford Press, New York.

Blomley N., Delaney D. and Ford R.T. (Eds), 2001, The legal geographies reader. Law, power, and space. Oxford, Blackwell Publishers.

Braverman I., 2011, "Hidden in Plain View: Legal Geography from a Visual Perspective", Law, Culture and the Humanities, volume 7, $\mathrm{n}^{\circ}$ 2, p. 173-186.

Braverman I., Blomley N., Delaney D., Kedar A. (eds), 2014, The Expanding Spaces of Law: A Timely Legal Geography, Stanford University Press, Standford.

Delaney D., 1993, "Geographies of Judgment: The Doctrine of Changed Conditions and the Geopolitics or Race ", Annals of the Association of American Geographers, volume 83, nº1, p. 48-65.

Forest P., 2009, Géographie du droit. Épistémologie, développement et perspectives, Presses de l'Université de Laval, Québec.

Holder J. \& Harrison C. (eds), 2003, Law and Geography, Oxford \& Toronto, Oxford University Press. Jones G. A., 2003, "Camels, Chameleons, and Coyotes: problematizing the 'Histories' of Land Law Reform' in Holder J. \& Harrison C. (eds), Law and Geography, Oxford \& Toronto, Oxford University Press, , p. 169-189 
Taylor W., 2006, The geography of law; landscape, identity and regulation, Oxford, Hart Publishing.

Thom B., 2004, "Geography, planning and the law : a coastal perspective", Australian Geographer, volume $35, \mathrm{n}^{\circ} 1$, p. 3-16.

\section{AUTEURS}

\section{NADIA BELAIDI}

Nadia Belaidi est chargée de recherche au CNRS (Section 39 Espaces, Territoires, Sociétés), UMR CNRS 7206 Eco-Anthropologie et Ethnobiologie. Elle est Chercheure en droit international et comparé de l'environnement, Nadia Belaidi travaille sur les perspective d'une justice environnementale via, notamment, la conceptualisation de la notion d'ordre public écologique et son expérimentation à partir de la démarche des Parcs pour la paix en Afrique Australe, nadia.belaidi@mnhn.fr

\section{GENEVIÈVE KOUBI}

Geneviève Koubi est professeur à l'université Paris 8 en droit public. Elle effectue ses recherches principalement dans le champ des sciences administratives, des droits sociaux et des sciences du texte appliquées au droit (circulaires administratives)CERSA-CNRS UMR, 7106, genevieve@koubi.fr 